\title{
COVID-19 and cardiac surgeon- Are we on the back-foot?
}

\author{
Sudhansoo Khanna ${ }^{1}$ \\ ${ }^{1}$ Post Graduate Institute of Medical Education and Research
}

April 28, 2020

\begin{abstract}
The coronavirus disease 2019 (COVID-19) is an infectious disease which has rapidly evolved into a pandemic. Though it has affected all disciplines of medical sciences but it has some serious implications pertaining to cardiovascular sciences which have presented unique challenges in front of cardiac surgeons in particular. To flatten the curve of this pandemic, routine cardiac surgeries are being deferred indefinitely resulting in the pool of sick cardiac patients rising day by day. A different perspective is presented on this global catastrophe from the viewpoint of a cardiac surgeon.
\end{abstract}

Text

Humanity is witnessing the Coronavirus disease 2019 (COVID-19) pandemic which no one ever imagined in their wildest of dreams. It is an evolving global threat and its future course appears to be unpredictable to say the least. As of April 18, 2020, there have been 20,78,605 confirmed cases of COVID-19 with 1,39,515 confirmed deaths reported worldwide and still counting. ${ }^{1}$

Although the exact clinical course, severity, and complications of COVID-19 are not yet completely determined but the risk of mortality is seen to be higher in males, age $>60$ years and those with underlying co-morbid conditions like diabetes mellitus, underlying cardiac or lung disorder, and any immunocompromised state. ${ }^{2}$ This fact is of critical importance for any healthcare provider dealing with cardiac sciences, more so for cardiac surgeons.

The exponential rate at which this situation is evolving has forced us to ask some critical questions about our preparedness.

This is an extraordinary situation which requires extra-ordinary measures. There is a global call for deferring the elective cardiac surgeries. The very step to postpone cardiac surgeries can be argued upon. There are three critical factors favoring deferring of such procedures. Firstly, it will help in the containment effort by reducing the travel of patient with accompanying relatives to the hospital which are hotspots for severe acute respiratory syndrome Coronavirus-2 (SARS-CoV-2). Secondly, reducing the number of elective procedures will preserve the essential medical supplies, intensive care unit (ICU) beds, ventilators, personal protective equipments (PPE), which can be utilized for dedicated COVID-19 units. Preserving protective equipment and clinicians is key in containing outbreak. Thirdly, cardiac surgeries utilize a large pool of blood components of the hospital and in this era of pandemic, the numbers of blood donors have reduced substantially. Thus, by avoiding elective cardiac surgeries, can help in preserving them for emergency cases

Coronaviruses are known to affect the cardiovascular system. Novel coronavirus, SARS-CoV-2 has been shown to affect the cardiovascular system by a number of mechanisms such as eliciting an intense systemic inflammatory response syndrome (SIRS), plaque instability and even direct cytotoxic effect on myocardium leading to myocarditis. ${ }^{3,4}$ It is well known that acute deterioration in lung function leads to increased cardiac workload and thus de-stabilizes underlying chronic cardiovascular diseases (CVDs). Therefore, SARS-CoV-2 affects the cardiac patients in a dual manner. Firstly, by causing severe acute respiratory syndrome (SARS), 
it creates extra workload on already diseased myocardium. Secondly, by inducing myocarditis it furthers depresses cardiac function. The decreased cardiac function in turn worsens lung function further, starting a vicious cycle in which SARS-CoV-2 acts as a catalyst, especially in cardiac patients.

Cardiopulmonary bypass (CPB) induces SIRS which is known to depress myocardial function and lung injury. ${ }^{5}$ Further it has been observed that SARS-CoV-2 is associated with a high inflammatory response. ${ }^{3}$ Thus, a nosocomial SARS-CoV-2 infection in a patient operated for an elective cardiac surgery under CPB can be lethal.

While there is a general consensus for postponement or delaying the elective cardiac surgeries, however defining elective cardiovascular procedures in the era of COVID-19 outbreak is not so straightforward. A number of guidelines are emerging globally highlighting various mechanism to triage cardiac patients requiring interventions. ${ }^{6}$ Each hospital should make their own standard set of guidelines which is best suited for their infrastructure and population to triage the cases.

Till the time a standard set of guidelines become available we recommend deferring the 'deferrable' cases. We believe performing percutaneous coronary interventions (PCI) and other endovascular procedures and resorting to surgical intervention only as a last ditch stand will be beneficial in the present scenario. While being on the back foot, for a cardiac surgeon, in this extraordinary situation of global pandemic makes more sense but at the same time we should work extensively towards preparing an exit strategy because although COVID-19 emerged as an acute infectious disease but it may soon become a chronic epidemic similar to influenza due to genetic recombination.

\section{Author contributions}

Dr. Sudhansoo Khanna - Concept/ design, Data collection, Data analysis, Critical revision of article, Drafting article.

\section{References}

1. WHO COVID-19 Dashboard. https://covid19.who.int/. Accessed April 18, 2020.

2. Fauci AS, Lane HC, Redfield RR. Covid-19: navigating the uncharted. N Engl J Med 2020. doi: 10.1056/NEJMe2002387

3. Madjid M, Safavi-Naeini P, Solomon SD, Vardeny O. Potential Effects of Coronaviruses on the Cardiovascular System: A Review [published online ahead of print, 2020 Mar 27]. JAMA Cardiol 2020;10.1001/jamacardio.2020.1286. doi:10.1001/jamacardio.2020.1286

4. Inciardi RM, Lupi L, Zaccone G, et al. Cardiac involvement in a patient with coronavirus disease 2019 (COVID-19). JAMA Cardiol. Published online March 27, 2020. doi:10.1001/jamacardio.2020.1096.

5. Westaby S. Complement and the damaging effects of cardiopulmonary bypass. Thorax 1983;38:321-325.

6. Driggin E, Madhavan MV, Bikdeli B, Chuich T, Laracy J, Bondi-Zoccai G, et al. Cardiovascular Considerations for Patients, Health Care Workers, and Health Systems During the Coronavirus Disease 2019 (COVID-19) Pandemic, Journal of the American College of Cardiology 2020, doi: https://doi.org/10.1016/j.jacc.20 